\title{
WHITE PATCH GAMUT MAPPING COLOUR CONSTANCY
}

\author{
Hamid Reza Vaezi Joze, Mark S. Drew \\ School of Computing Science, Simon Fraser University \\ Vancouver, BC, Canada V5A 1S6 \\ $\{$ hrv1,mark\}@cs.sfu.ca
}

\begin{abstract}
The White-Patch method, one of the first colour constancy methods, estimates the light source colour from the maximum response of the different colour channels. However, it has been eclipsed by the advent of more advanced physical or statistical methods, as well as complex learning based methods. Recently, a new independent line of work claims that the simple idea of using maximum pixel values is not as naive as it seems, but can also be made to perform very well via some manipulations. The bright areas of images can include highlights and specularity as well as white surfaces or light sources, and indeed all may be helpful in the illumination estimation process.

In this paper, we define the White Patch Gamut as a new extension to the Gamut Mapping Colour Constancy method, comprising the bright pixels of the image. Adding new constraints based on the possible White Patch Gamut to the standard gamut mapping constraints, a new combined method outperforms gamut mapping methods as well as other wellknown colour constancy methods. The new constraints that are brought to bear are powerful, and indeed can be more discriminating than those in the original gamut mapping method itself.
\end{abstract}

Index Terms - Colour Constancy, Gamut Mapping, Illumination Estimation.

\section{INTRODUCTION}

Estimation of illumination, the main concern of colour constancy processing, is an important problem in image processing for digital still images or video, forming a prerequisite for many computer vision applications. The foundational colour constancy method, the so-called White-Patch or Max-RGB method estimates the light source colour from the maximum response of the different colour channels [1]. With the advent of newer colour constancy methods such as Grey-World [2], Gamut Mapping [3], Grey-Edge [4] and many other complex algorithms (see [5] for an overview), few researchers in the field or commercial cameras use the White-Patch method. On the other hand, recent works by Choudhury and Medioni [6] and Funt and Shi [7] proposed that finding the maximum after a local mean calculation such as local blurring can significantly improve the performance of this simple method. Moreover, Drew et al. [8] analytically showed that the geometric mean of pixels in bright (generally, specular) pixel regions is the optimal estimate for the illuminant. This insight says that in the presence of specular highlights illuminant estimation is the mean of the max, while former works $[7,6]$ argued the converse. The analytical approach [8] claims comparable performance with complex colour constancy methods despite its simple approach.

The Gamut Mapping algorithm, a more complex and more accurate algorithm, was introduced by Forsyth [3]. It is based on the assumption that in real-world images, for a given illuminant one observes only a limited number of colours. Several extensions have been proposed $[9,10,11,12]$.

The bright areas of images can be highlights and specularity as well as white surfaces or light sources, and all are helpful in the illumination estimation process. Highlights and white surfaces both tend to have the colour of light in ideal conditions.

In this paper, we define the White Patch Gamut as a new extension to the Gamut Mapping Colour Constancy method, consisting of the gamut of the bright pixels of an image. New constraints are added as a result. Experiment shows that adding these new constraints to those from standard gamut mapping constraints outperforms gamut mapping methods as well as other well-known colour constancy methods.

\section{GAMUT MAPPING}

Forsyth's [3] gamut mapping algorithm is one of the most successful colour constancy algorithms. Its strength lies in the fact that it makes the uncertainty in the illuminant explicit. It is based on the assumption that in real-world images, for a reference illuminant, one observes only a limited number of colours. Consequently, any variations in the colours of an image is due to variation in the colour of light. The convex hull of limited set of $R G B \mathrm{~s}$ that can occur under a reference illuminant is called the canonical gamut, denoted $\mathcal{C}$. The canonical gamut is found in a training phase by observing surfaces under known light sources.

In general, a gamut mapping algorithm accepts an image captured under an unknown light source and generates the input gamut $\mathcal{I}$ using the set of $R G B$ values in the input image, and then determines the set of feasible mappings $\mathcal{M}$, consisting of all possible mappings that can map the input gamut to lie completely within the canonical gamut. Finally an esti- 
mator is needed to select one mapping from the set of feasible mappings. The selected mapping can be applied to the canonical illuminant to obtain an estimate of the unknown illuminant. Assuming $R G B$ values are linear responses to the camera sensors, These mappings mostly have been modeled by a diagonal mapping, which is a diagonal matrix that maps image colours under an unknown light source $i$ to their corresponding colours under the canonical illuminant $c$.

$$
\rho=\left(\begin{array}{c}
R_{c} \\
G_{c} \\
B_{c}
\end{array}\right)=D\left(\begin{array}{c}
R_{i} \\
G_{i} \\
B_{i}
\end{array}\right) \quad D=\left(\begin{array}{ccc}
d_{1} & 0 & 0 \\
0 & d_{2} & 0 \\
0 & 0 & d_{3}
\end{array}\right)
$$

The estimator to select one mapping could be the diagonal matrix with the largest trace, as originally suggested [3], or the average or weighted average of the feasible set [9].

Finlayson and $\mathrm{Xu}$ [13] introduced an efficient implementation of gamut mapping using convex programming. In this implementation the canonical gamut is rewritten in terms of $N$ inequalities which represent the 3-dimensional convex hull of the set of $R G B \mathrm{~s}$ in the canonical gamut:

$$
\mathcal{C}(\rho): \quad \begin{gathered}
a_{1} R+b_{1} G+c_{1} B \\
a_{2} R+b_{2} G+c_{2} B \geq e_{1} \\
\vdots \\
a_{N} R+b_{N} G+c_{N} B \geq e_{N}
\end{gathered}
$$

Defining an $N \times 3$ matrix $A$ with $i$ th row equal to $\left[a_{i} b_{i} c_{i}\right]$ and an $N \times 1$ vector $e$, with $i$ th component equal to $e_{i}$, we can rewrite this formulation as follows:

$$
\mathcal{C}(\rho): A \rho \geq e
$$

Now using the diagonal transform we have $\rho=q \operatorname{diag}(d)$ or equally $\rho=\operatorname{diag}(q) d$, where $d$ is $\left[d_{1}, d_{2}, d_{3}\right]$ and $q$ is the $R G B$ colours of the image under the unknown light source. If we have $K 3$-dimensional points in the convex hull of input gamut $\mathcal{I}$ for each image, there will be $N \times K$ linear constraints for each image, which defines possible mappings:

$$
\mathcal{M}: \forall q_{i} \in \mathcal{I}: A \operatorname{diag}\left(q_{i}\right) d \geq e
$$

In order to estimate the illuminant an optimality criterion must be defined, such as maximizing the $\mathrm{L}_{1}$ norm of the diagonal.

\section{WHITE PATCH GAMUT MAPPING}

The foundational color constancy method, so called White Patch method, usually estimates the illuminant by finding a white patch in the scene which has maximum response in all channels. Experiments show that illuminant information is more informative in bright regions than darker region $[14,15]$. Let us extend white patch to assume that there is always a white patch, highlights or light source in the image. We also use the gamut of bright pixels, in contradistinction to WhitePatch, which usually deals only with the brightest pixel in the image. Obviously, using a single pixel can be noisy and is not robust.

Since we dealing with bright pixels we need to be careful about clipped pixels, which are pixels where the light reflection exceeds the dynamic range of the camera. We remove pixels which exceed $90 \%$ of the dynamic range for each channel. Then we simply define bright pixels as the top $T \%$ of the sum of channels $R+G+B$. Here, $T$ could be 1,2 or $5 \%$.

To investigate the utility of this assumption, we carry a simple experiment to check whether the actual illuminant colour falls inside the 2D gamut of bright pixels or not. We find that the actual illuminant colour falls in the 2D gamut of the top 5\% brightness pixels of each image for SFU Laboratory Dataset [16] for $88.16 \%$ of images, and in $66.02 \%$ of images for the GreyBall Dataset [17]. Fig. 4 shows 2D gamut in chromaticity space $\{r, g\}=\{R, G\} /(R+G+B)$, with the top $5 \%$ brightness pixels in green. The actual measured illuminant is shown as a red star. Note that with no supporting evidence (Fig. 4(c)) the illuminant may not fall in the bright region.
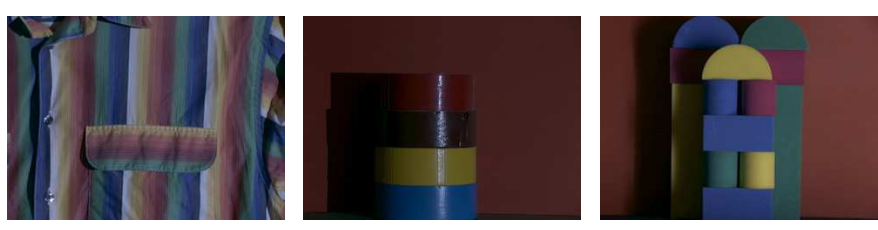

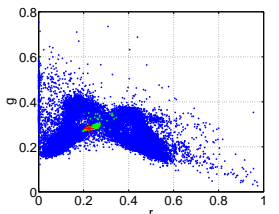

(a)

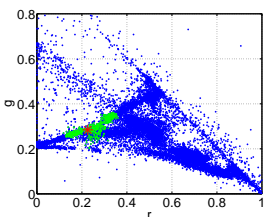

(b)

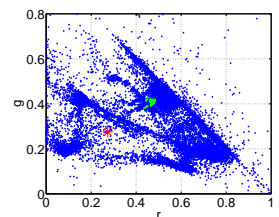

(c)
Fig. 1. Examples of image evidence: top 5\% bright pixels in green, other pixels in blue, and red star showing the illuminant point in $r, g$ chromaticity space. (a) Image with white patch; (b) Image with specularity; (c) Image without white patch or specularity.

Motivated by this experiment, we define the White Patch Gamut, consisting of the set of $R G B$ s in an image for a white patch, highlights, or light source. As well, we define the canonical White Patch Gamut, denoted $\mathcal{C}_{w}$, comprising the limited set of $R G B$ s that can occur for a white patch, highlights, or light source under a reference illuminant. This can be trained by observing images under known light sources. I.e., we simply identify them in a training phase by collecting the top-brightness pixels which fall near actual values of the illuminant colour, projected onto 2D chromaticity space. Respectively, input White Patch Gamut $\mathcal{I}_{w}$ is defined as the gamut of the top-brightness pixels for an input image.

$$
\mathcal{C}_{w}(\rho): \begin{gathered}
a_{1}^{w} R+b_{1}^{w} G+c_{1}^{w} B \geq e_{1}^{w} \\
a_{2}^{w} R+b_{2}^{w} G+c_{2}^{w} B \geq e_{2}^{w} \\
\vdots \\
\\
a_{N}^{w} R+b_{N}^{w} G+c_{N}^{w} B \geq e_{N}^{w}
\end{gathered}
$$

We can again define $A_{w}$ and $e_{w}$ in the same fashion as $A$ and $e$. Using the principle of gamut mapping set out in section 2 , we can determine the set of feasible mappings $\mathcal{M}_{w}$, meaning all possible mappings that can transfer the input White 
Patch Gamut to lie completely within the canonical White Patch Gamut.

$$
\mathcal{M}_{w}: \forall q_{i} \in \mathcal{I}_{w}: A_{w} \operatorname{diag}\left(q_{i}\right) d \geq e_{w}
$$

These constraints are only in operation if there is a white patch or specularity in the image. However our experiments show that, if they can indeed be brought into play, these new constraints are even more powerful than the original gamut mapping constraints.

\subsection{Generating the White Patch Gamut}

When there are no strong highlights, light sources, or white surfaces in the image, the bright pixels are not helpful; in such a case they can stem from areas of images belonging to the brightest surface, which tends to be some particular surface colour or possibly a bag of single pixels from all over the image. In the former case we can simply check if these pixels are in the possible chromaticity gamut of illuminants (this gamut is computed in the training phase) and in the latter we can investigate the distribution of these pixels in chromaticity space. The standard deviation of these pixels in RGB space is a good measure for this purpose. In either case, there is no White Patch Gamut for such an image. Thus, we cannot necessarily define a White Patch Gamut for all images. On the other hand, in order to omit outliers we remove pixels with distance to the mean greater than 2 standard deviations. Fig. 2 shows (red) the 2D gamut and the white patch gamut for example images, compared to their canonical gamuts (blue) in chromaticity space.
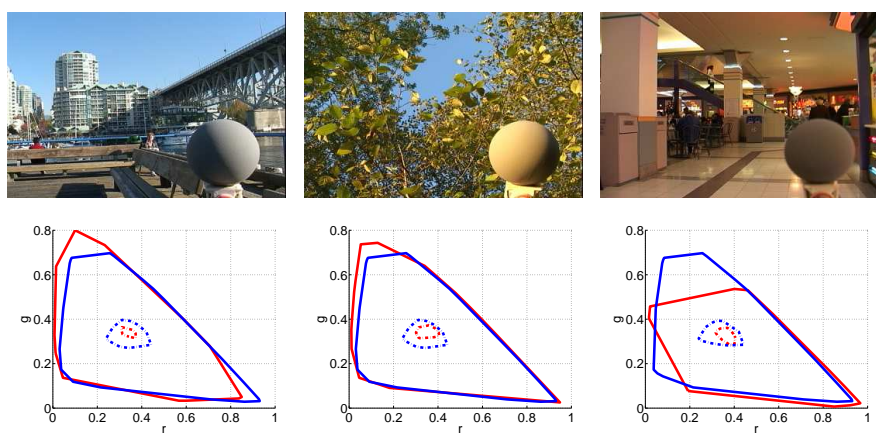

Fig. 2. Examples of 2D gamut (solid red) and White Patch Gamut (dotted red) compared to canonical gamut (solid blue) and canonical White Patch Gamut (dotted blue) in $r, g$ chromaticity space.

Fig. 3 shows the 3D canonical gamut for the SFU Laboratory dataset and its 3D canonical White Patch Gamut in $R G B$ space (refer to $\S 4$ for more detail). As we expected the White Patch Gamut is smaller.

\subsection{Combination Method}

Until now, we have two sets of feasible mappings: $\mathcal{M}$, which is based on standard gamut mapping and $\mathcal{M}_{w}$, which is based on white patch gamut mapping. These sets can be used in combination instead of selecting only one mapping per algorithm. Since each feasible set represents all illuminant estimates that are considered possible, an obvious combination is to consider only those estimates that are all feasible sets, which is intersection of these two sets $\left(\mathcal{M} \cap \mathcal{M}_{w}\right)$. Another approach to combine these is to consider all possible estimates in both feasible sets, meaning the union of these two sets $\left(\mathcal{M} \cup \mathcal{M}_{w}\right)$. Experiment shows that the intersection approach outperforms the union approach. For the intersection based combination, the optimization becomes the following convex programming problem:

$$
\begin{array}{rc}
\text { Maximize } & d_{1}+d_{2}+d_{3} \\
\text { subject to } & A \operatorname{diag}(q) d \geq e, q \in I \\
& A_{w} \operatorname{diag}(q) d \geq e_{w}, q \in I_{w}
\end{array}
$$

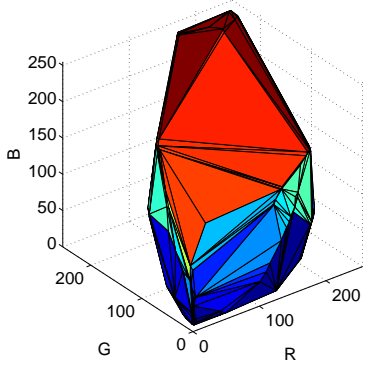

(a)

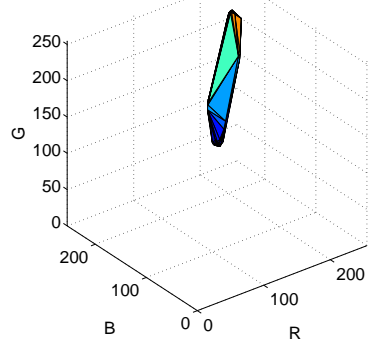

(b)
Fig. 3. (a) 3D canonical gamut (b) 3D canonical white patch gamut for SFU Laboratory dataset in $R G B$ colour.

\section{EXPERIMENTAL RESULTS}

First, consider the Barnard colour constancy dataset [16], which we call the SFU Laboratory dataset; this contains 321 measured images under 11 different measured illuminants. The scenes are divided into two sets as follows: minimal specularities (223 images), and non-negligible dielectric specularities (98 images).

For a more real-world (out of the laboratory) image experiment we also used the Ciurea and Funt [17] GreyBall dataset, which contains 11346 images extracted from video recorded under a wide variety of imaging conditions. The images are divided into 15 different clips taken at different locations. The ground truth was acquired by attaching a grey sphere to the camera, displayed in the bottom-right corner of the image. This grey sphere must be masked out during experiments.

We use Matlab code provided by [12] for general gamut mapping, which is based on convex programming. For the SFU Laboratory dataset, 31 images (all images recorded under the syl-50MR16Q-illuminant) were used for computation of the canonical gamut, and these were omitted from the test set. For the GreyBall Dataset, the canonical gamuts were computed using 14 out of 15 categories and we tested on the 
Table 1. Comparison of White Patch Gamut Mapping (WP) with well known colour constancy methods.

\begin{tabular}{|l|cc|cc|}
\hline Dataset & \multicolumn{2}{|c|}{ SFU Labratory } & \multicolumn{2}{c|}{ GreyBall } \\
Methods & Median & Mean & Median & Mean \\
\hline White Patch & $6.5^{\circ}$ & $9.1^{\circ}$ & $5.3^{\circ}$ & $6.8^{\circ}$ \\
Grey World & $7.0^{\circ}$ & $9.8^{\circ}$ & $7.0^{\circ}$ & $7.9^{\circ}$ \\
Grey Edge & $3.2^{\circ}$ & $5.6^{\circ}$ & $4.7^{\circ}$ & $5.9^{\circ}$ \\
Gamut Mapping & $2.3^{\circ}$ & $3.9^{\circ}$ & $5.5^{\circ}$ & $7.0^{\circ}$ \\
Gamut Mapping 1-jet & $2.1^{\circ}$ & $3.9^{\circ}$ & $5.5^{\circ}$ & $6.8^{\circ}$ \\
Natural Image Stat. & - & - & $\mathbf{3 . 9}^{\circ}$ & $\mathbf{5 . 2}^{\circ}$ \\
WP Gamut Mapping & $\mathbf{1 . 9}^{\circ}$ & $\mathbf{3 . 4}^{\circ}$ & $4.7^{\circ}$ & $6.0^{\circ}$ \\
\hline
\end{tabular}

remaining category. We repeated the experiment 15 times. We used the top $5 \%$ of the sum of channels to generate the bright gamut.

Table 1 gives the accuracy of the proposed methods for the SFU Laboratory dataset [16] as well as the GreyBall dataset [17], in terms of the mean and median of angular errors, for several colour constancy algorithms applied to this dataset. For those methods which need tunable parameters, we utilize optimal parameters for each dataset. (In these experiments, we actually accomplished slightly better estimation for pixel based gamut mapping than other reported results for the GreyBall dataset since we mask image margins because of their low quality.)

This experiment shows that white patch gamut mapping improves the performance of gamut mapping by $13 \%$ to $18 \%$ in terms of angular mean and median error; these results show that the proposed method is in fact the best extension of gamut mapping compared to other extensions such as generalized edge-based gamut mapping [12] (1st-jet is their best) or the 2D version of gamut mapping [11]. To our knowledge, for the SFU Laboratory dataset white patch gamut mapping does best in terms of both mean and median angular error compared to any reported colour constancy method, even very complex ones. For the GreyBall dataset, for which gamut mapping generally does not work very well (since the images are not gamma corrected), white patch gamut mapping outperforms gamut mapping by $15 \%$; it does better than or equal to all the unsupervised colour constancy methods, but does not surpass those which combine or select other colour constancy methods, such as Natural Image Statistics [18].

\section{CONCLUSION}

In this paper, we define the White Patch Gamut as a new extension to the gamut mapping colour constancy method, consisting of the bright pixels of the image. Our experiments show that adding new constraints based on the White Patch Gamut to standard gamut mapping constraints outperforms gamut mapping methods as well as other well-known colour constancy methods. These new constraints are therefore a powerful addition to the field and in fact can be more discriminating than the original set of constraints themselves.

White patch gamut mapping improves the performance of gamut mapping by $13 \%$ to $18 \%$ in terms of angular mean and median error over the two well-known standard datasets that we tested, thus comprising is the best extension of gamut mapping colour constancy to date.

\section{REFERENCES}

[1] E. Land, "The retinex theory of color vision," Scientific American, vol. 237, no. 6, pp. 108-128, Dec. 1977.

[2] G. Buchsbaum, "A spatial processor model for object colour perception,” J. Franklin Inst., vol. 310, pp. 1-26, 1980.

[3] D.A. Forsyth, "A novel approach to color constancy," in Int. Conf. on Computer Vision '88, 1988, pp. 9-18.

[4] J. van de Weijer and T. Gevers, "Color constancy based on the grey-edge hypothesis," in Int. Conf. on Image Proc., 2005, pp. II:722-725.

[5] S. D. Hordley, "Scene illuminant estimation: past, present, and future," Color Research and Application, vol. 31, no. 4, pp. 303-314, 2006.

[6] A. Choudhury and G. Medioni, "Perceptually motivated automatic color contrast enhancement," in Color and Reflectance in Imaging and Comp. Vis. Workshop, 2009, pp. 1893-1900.

[7] B. Funt and L. Shi, "The rehabilitation of maxrgb," in 18th Color Imaging Conference, 2010, pp. 256-259.

[8] M. Drew, H. Vaezi Joze, and G. Finlayson, "Specularity, the zeta-image, and information-theoretic illuminant estimation," in Tech. Rep. TR 2012, Simon Fraser University, 2012.

[9] K. Barnard, "Improvements to gamut mapping colour constancy algorithms," in European conference on computer vision, 2000, pp. 390-403.

[10] G. Finlayson and S. Hordley, "Improving gamut mapping color constancy," IEEE Transactions on Image Processing, vol. 9, no. 10, pp. 1774-1783, 2000.

[11] G.D. Finlayson, "Color in perspective," IEEE Trans. Patt. Anal. and Mach. Intell., vol. 18, pp. 1034-1038, 1996.

[12] A. Gijsenij, T. Gevers, and J. van de Weijer, "Generalized gamut mapping using image derivative structures for color constancy," Int. J. Comput. Vision, vol. 86, pp. 127-139, 2008.

[13] G. D. Finlayson and R. Xu, "Convex programming color constancy," in IEEE workshop on color and photometric methods in computer vision, Int. Conf. on Comp. Vision, 2003.

[14] S. Tominaga, S. Ebisui, and B.A. Wandell, "Scene illuminant classification: brighter is better," J. Opt. Soc. Am. A, vol. 18, pp. 55-64, 2001.

[15] C. Fredembach and G. D. Finlayson, "Bright chromagenic algorithm for illuminant estimation," The Journal of Imaging Science and Technology, vol. 52, no. 4, pp. 1-11, 2008.

[16] K. Barnard, L. Martin, B. Funt, and A. Coath, "A data set for colour research," Color Research and Applications, vol. 27, pp. 147-151, 2002.

[17] F. Ciurea and B. V. Funt, "A large image database for color constancy research," in IS\&T/SID Color Imaging Conference, 2003, pp. 160-164.

[18] A. Gijsenij and T. Gevers, "Color constancy using natural image statistics and scene semantics," IEEE Trans. Patt. Anal. and Mach. Intell., vol. 33, no. 4, pp. 687-698, 2011. 